\title{
Guía de campo del complejo México-Querétaro-Hidalgo, centro de México
}

Luis Miguel Espinosa Rodríguez.

geo_luismiguel@hotmail.com

Facultad de Geografía, UAEMéx

Recibido:29-noviembre -2019

Aceptado:24-enero-2020

\section{Resumen}

El trabajo de campo en la carrera de Geografía en cualquier universidad del mundo es imprescindible desde la perspectiva disciplinaria y pedagógico-didáctica. El binomio gabinete-campo resulta indisoluble cuando los objetivos centrales de un plan de estudios se enfocan en formar profesionales en el ámbito geográfico. De acuerdo con ello, en el presente trabajo se expone una guía de campo para la asignatura de Geografía del paisaje que se imparte en la Licenciatura en Geografía de la Universidad Autónoma del estado de México. Se muestran las características y objetivos del programa de estudios y la concepción metodológica que sustenta al curso y a la práctica de campo, para con ello exhibir un recorrido por los estados de México, Hidalgo y Querétaro en donde se instrumentan técnicas y metodologías para alcanzar los objetivos de aprendizaje.

Palabras clave: trabajo de campo, Geografía, guía, paisaje, aprendizaje.

\section{Mexico-Queretaro-Hidalgo complex field guide, central Mexico}

\begin{abstract}
The field work in the career of Geography in any university in the world is essential from the disciplinary and pedagogical-didactic perspective. The cabinet-field binomial is indissoluble when the central objectives of a curriculum are focused on training professionals in the geographical area. Accordingly, in this paper a field guide for the subject of Landscape geography is taught in the Bachelor of Geography of the Autonomous University of the State of Mexico. The characteristics and objectives in the study program and the methodological conception that supports the course and the field practice are show, to thereby exhibit a tour of the states of Mexico, Queretaro and Hidalgo where techniques and methodologies are implemented to achieve the objectives learning.
\end{abstract}

Key words: work field, Geography, guide, landscape, 


\section{Introducción}

La práctica para la unidad de aprendizaje "Geografía del Paisaje" se encuentra sustentada en los objetivos del Plan de Estudios F de la Licenciatura en Geografía que se imparte en la Facultad de Geografía de la Universidad Autónoma del Estado de México. De forma administrativa, el área curricular a la que pertenece la asignatura es la de Ciencias de la Tierra, la cual se imparte en una modalidad escolarizada que integra seis horas semanales de trabajo desagregadas en una teórica y cinco prácticas; las cuales otorgan a los estudiantes adscritos a la asignatura, seis créditos en el historial académico.

El objetivo de la Unidad de aprendizaje refiere textualmente a: "Evaluar en campo los subsistemas físicos, biológicos y humanos como componentes diferenciadores $e$ integradores del paisaje y los procesos que se manifiestan en él, para estructurar propuestas de ordenación del territorio con apoyo de herramientas geotecnológicas, en congruencia con el manejo óptimo y sostenible de sus potencialidades".

El curso ha sido planeado con el propósito de cubrir cuatro objetivos particulares; el primero de ellos, titulado "Introducción al análisis metodológico del territorio" tiene como objetivo desarrollar habilidades de pensamiento para el estudio del territorio a través del análisis del espacio geográfico entendido como un sistema para el entendimiento del espacio geográfico.

La segunda unidad de trabajo se denomina "Metodologías para el análisis territorial a través de la Geografía del Paisaje"; en la cual se propone analizar métodos y técnicas desarrolladas por diferentes escuelas de pensamiento, con la finalidad de contar con criterios que permitan seleccionar la que mejor se adapte para el desarrollo de un estudio de caso.

El tercer apartado del programa de estudios se denomina "Cartografía de paisaje y su interpretación", en el cual se elaborar cartografía de unidades de paisaje aplicada a una investigación geográfica mediante el uso de una metodología definida con la finalidad de interpretar la dinámica y evolución de las unidades de paisaje y su análisis en campo. 
Finalmente, el último segmento de aprendizaje es intitulado "Aplicación de la Geografía del Paisaje en el análisis de problemas espaciales en distintos ámbitos de la geografía"; y tiene como propósito analizar la importancia de la geografía del paisaje en proyectos de análisis espacial mediante aplicaciones realizadas en diferentes ámbitos de la geografía.

El enfoque y los principios pedagógicos que guían el desarrollo de la unidad de aprendizaje se basan en la corriente constructivista del aprendizaje y la enseñanza, según la cual el aprendizaje es un proceso constructivo interno que realiza el estudiante a partir de su actividad interna y externa y, por intermediación del profesor -facilitador-, que propicia diversas situaciones de aprendizaje para facilitar la construcción de aprendizajes significativos y contextualizar el conocimiento. Por tanto, los métodos, estrategias y recursos de enseñanza - aprendizaje está enfocada a cumplir los siguientes principios:

- El uso de estrategias motivacionales para influir positivamente en la disposición de aprendizaje de los estudiantes.

- La activación de los conocimientos previos de los estudiantes a fin de vincular lo que ya sabe con lo nuevo que va a aprender.

- El diseño de diversas situaciones y condiciones que posibiliten diferentes tipos de aprendizaje.

- La propuesta de diversas actividades de aprendizaje que brinden al estudiante diferentes oportunidades de aprendizaje y representación del contenido.

En conformidad con lo anterior, y con la intención de facilitar el aprendizaje de los contenidos y lograr los objetivos educativos, se diseñó una metodología de enseñanza centrada en el aprendizaje, para lo cual en cada una de las secuencias didácticas que integran una guía de aprendizaje en la cual se incluyeron diferentes actividades de enseñanza para que el estudiante tenga oportunidad de integrar, practicar o transferir los conocimientos adquiridos en cada unidad temática en gabinete y en campo.

De acuerdo con lo anterior, se interpreta que el programa incide en el estudio particular de metodologías que permiten el desarrollo de una evaluación holística del territorio y por ello se trabaja para cumplir con objetivos de integración, pues se parte del supuesto en el cual los alumnos adscritos al curso se encuentran preparados para aplicar los 
conocimientos que alcanzaron a lo largo del curso y en general, los adquiridos hasta el momento en su transcurso por la Facultad de Geografía.

Así, el trabajo en campo permite la aplicación de la base teórica y metodológica para abordar con claridad los problemas de la envoltura geográfica como las regularidades de la diferenciación local y/o topológica de la envoltura geográfica, así como de los complejos naturales, la dinámica, desarrollo y funcionamiento.

En este sentido, los propósitos de la práctica de campo se diseña de acuerdo con los propósitos generales observados en el Plan de Estudios vigente de tal forma que alumno será competente en: la definición de las condiciones del fenosistema y el criptosistema; en la delimitación de las unidades territoriales; en la diferenciación de los tipos y las características de los componentes territoriales bióticos, abióticos, socioeconómicos y paisajísticos; y finalmente en el desarrollo de métodos de regionalización y análisis morfoedáfico.

\section{Metodología}

En cada parada de trabajo se propició el análisis geográfico regional y local de los sitios, incluyendo aspectos geológicos, geomorfológicos, climáticos, edáficos y bióticos; se ha realizado la caracterización general del relieve y del paisaje en función de variables de latitud y altitud; zonalidad, azonalidad, así como el balance de sustancias (materia y energía) entre otros; y queda claro, que se destaca la observación, análisis y aplicación de los elementos básicos del análisis geográfico: observación, localización, causalidad, distribución espacial e integración.

En este orden de ideas, se aplican los modelos de evaluación de paisaje sugeridos por diferentes escuelas de pensamiento del paisaje como la mexicana, cubana, española, francesa y soviética entre otras; así como los derivados de la metodología morfoedáfica desarrollada en nuestro país.

En primer término, el análisis previo de paisaje requiere de clarificar aspectos visuales (que requieren de comprensión, análisis cualitativo y cuantitativo) de aspectos referentes a 
los mosaicos más o menos ordenado de formas y colores; a la consistencia sólida, líquida de los elementos observados; a la diferenciación de los elementos dotados de vida y/o inanimados; y a las diferencias acusadas que mantienen un orden en el espacio, no estáticas. Todo ello para comenzar una serie de procesos cognitivos relacionados con diferentes fases de complejidad como:

a. La distinción de la heterogeneidad y la homogeneidad.

b. El análisis de los elementos en función de su forma y magnitud

c. La clasificación de los paisajes (morfológicos, de vegetación, agrarios, agrícolas, etcétera)

Por otra parte, en cada sitio visitado, se determinan las condiciones particulares de los fenosistemas y de los cripsotistemas a través de la observación -y parametrización, si procede- de elementos representativos del paisaje y de la geomorfología del lugar a través del empleo de diversas variables como son:

- $\quad$ Estructuras geológicas

- $\quad$ Tipos de rocas

- $\quad$ Orientación general de espejo de falla principal

- $\quad$ Dirección general del río principal

- $\quad$ Número de celdas solares en la región

- $\quad$ Tipos de árboles

- Indicadores de contaminación

- $\quad$ Número y niveles de terrazas fluviales

- $\quad$ Localización de las zonas de sedimentación

- $\quad$ Número de sistemas fluviales en bifurcación con el sistema principal

- $\quad$ Definición y caracterización de los sistemas torrenciales

- $\quad$ Características distintivas de las laderas (orientación)

- $\quad$ Número de zonas con derrumbes

- $\quad$ Tipo predominante de suelo

- $\quad$ Número de establecimientos comerciales

- $\quad$ Presencia de epífitas

Por otra parte, según se avanza en el recorrido, se realizan hipótesis y se contrastan conceptos de: heterogeneidad y homogeneidad en relación con la escala; complejidad y globalidad de 
las formas de la superficie terrestre; y sobre la estructura y organización de la superficie terrestre. La finalidad de disertar y argüir en este tenor, lleva a los estudiantes a la aplicación de los métodos de estudio de los Complejos territoriales naturales (CTN), destacándose entre otras particularidades:

- $\quad$ El complejo de miembros.

- $\quad$ La estructura espacial diferente (complejidad y nivel sistémico de integración).

- $\quad$ La búsqueda de regularidades espaciales.

a. El empleo y aplicación correcta de expresiones metodológicas (geobiocenosis, cartográfica, geofísica, matemática, biogeográficas y paleogeográfica entre otras)

De acuerdo con los autores clásicos como Mateo (1984), De Bolós (1991) y Ortiz (2019) entre otros, se realizan ejercicio de introducción a la clasificación definiendo conceptos:

Sistematización: Agrupación de un conjunto de objetos en relación con determinadas semejanzas, ordenándolos de acuerdo con ellas y disponiéndolos de tal forma que el rango inferior se refiera al superior al igual que la parte al todo.

Clasificación: División de un conjunto en grupos de objetos homogéneos de acuerdo con las normas establecidas en la sistematización; supone la división horizontal de objetos similares de acuerdo con su rango. No se pueden reunir objetos diversos, heterogéneos, como por ejemplo los tipos de vegetación con las formas del relieve.

Taxonomía: División "vertical” de los objetos, en tanto que toma en cuenta su jerarquía y su subordinación. Crea posibilidades de clasificación en muchos niveles: un ejemplo de taxonomía lo tenemos en la clasificación de los paisajes por tamaño.

Tipificación: Consiste en hacer una lista, con unos criterios fijados de antemano, es decir, no de todos los objetos posibles a un nivel dado, sino tan sólo de aquellos que se encuentran en un determinado grupo o área considerada. Se tipifican los paisajes en naturales o artificiales, de acuerdo con la energía

Clasificación de los paisajes con relación a su estado morfoedáfico: 
Paisajes en equilibrio: cuando las entradas y salidas de materia y energía son parecidas, estables y la erosión es mínima (paisajes en biostasia); o de rexistasia cuando las variables se invierten.

Con los resultados obtenidos, se realiza la evaluación de los paisajes de acuerdo con los criterios de D’Luna (1995) quien clasifica a los paisajes en tres estadíos particulares (Tabla 1):

\begin{tabular}{c|ll}
\hline \multicolumn{2}{c}{ Clasificación de paisajes } \\
\hline Tipología & \multicolumn{2}{c}{ Características generales } \\
\hline \multirow{3}{*}{ Optimizados } & \multicolumn{2}{c}{ actividad humana mínima. } \\
& - & Provoca bajo o nulo impacto al medio natural. \\
& - & Se encaminan a la preservación y conservación del medio. \\
\hline & - & Actividades económicas que no provocan gran alteración. \\
Compensados & - & Insumos naturales \\
& - & Sustitución de cubierta vegetal por cultivos o vegetación \\
& equivalente. \\
\hline \multirow{3}{*}{ Agotados } & - & Actividades humanas que a gran escala han causado impacto. \\
& - & Abatimiento de agua, suelo y biota. \\
& - & cambios en la estructura horizontal. \\
\hline \multirow{2}{*}{ Alterados } & - & Actividades de gran impacto. \\
& - & Desaparición de uno o varios componentes. \\
\hline
\end{tabular}

Tabla 12. Sistema de clasificación de los paisajes de acuerdo con el "estado" que guardan. Fuente: D’Luna, 1995.

Por otra parte, se realiza en los últimos sectores de la visita a campo la determinación de la degradación de paisaje con base en la tabla de Bilwitz (1980):

\begin{tabular}{l|c|l|}
\hline \multicolumn{3}{c}{ Clasificación de los geosistemas transformados antropogenéticamente } \\
\hline $\begin{array}{l}\text { Grado de } \\
\text { transformación }\end{array}$ & $\begin{array}{l}\text { Grado de } \\
\text { hemerobia del } \\
\text { geosistema }\end{array}$ & $\begin{array}{l}\text { Procesos e índices del impacto } \\
\text { antropogénico y tecnogenético }\end{array}$ \\
\hline $\begin{array}{l}\text { No existen } \\
\text { transformaciones }\end{array}$ & Ahemeróbico & No existe impacto tecnogénico. \\
\hline $\begin{array}{l}\text { Transformaciones } \\
\text { reversibles de las } \\
\text { propiedades }\end{array}$ & Oligohemeróbico & $\begin{array}{l}\text { Débil utilización de los bosques y pastos. } \\
\text { Contaminación de las tierras por aguas } \\
\text { residuales y sustancias dañinas contenidas en } \\
\text { el aire. }\end{array}$ \\
\hline
\end{tabular}




\begin{tabular}{|c|c|c|}
\hline $\begin{array}{l}\text { potenciales } \\
\text { geosistemas }\end{array}$ & Mesohemeróbico & $\begin{array}{l}\text { Utilización pastoril de las tierras. Uso de los } \\
\text { bosques. Intensa utilización turística. }\end{array}$ \\
\hline \multirow{2}{*}{$\begin{array}{l}\text { Transformaciones } \\
\text { reversibles de las } \\
\text { propiedades laterales y } \\
\text { variables de los } \\
\text { geosistemas }\end{array}$} & \multirow[t]{2}{*}{ Oihemeróbico } & $\begin{array}{l}\text { Laboreo de suelo. Intenso uso no agrícola y } \\
\text { pastoril de las tierras. Forestación de las } \\
\text { ciudades. Creación de macizos arbóreos. } \\
\text { Reforestación artificial en correspondencia } \\
\text { con las condiciones ecológicas. }\end{array}$ \\
\hline & & $\begin{array}{l}\text { Formación de huecos. Hidromorfización } \\
\text { antropogénica de los suelos profundos y } \\
\text { fértiles para la horticultura. }\end{array}$ \\
\hline \multirow{2}{*}{$\begin{array}{l}\text { Transformaciones } \\
\text { irreversibles de las } \\
\text { propiedades } \\
\text { establecidas de } \\
\text { geosistemas }\end{array}$} & Polihemeróbico & $\begin{array}{l}\text { Creación de superficie parcial o débilmente } \\
\text { permeable. Rellenos por basamentos } \\
\text { artificiales y basureros. Extracción o relleno } \\
\text { de suelos fértiles. } \\
\text { Introducción parcial de elementos tóxicos en } \\
\text { la circulación substancial. }\end{array}$ \\
\hline & Metahemeróbico & $\begin{array}{l}\text { Creación de una superficie abierta } \\
\text { impermeable. Envenenamiento del suelo. } \\
\text { Afloramiento, liquidación y enterramiento } \\
\text { de los suelos y basamento. }\end{array}$ \\
\hline
\end{tabular}

Tabla 2. Sistema de clasificación de los geosistemas transformados por el hombre. Fuente: Bilwitz, 1980.

Una vez realizada la clasificación de estados de los paisajes y determinada la transformación de los paisajes, se realizan ejercicios relacionados con diferentes enfoques:

a. Basados en De Bolós (1991), se determinan condiciones de diagnosis (de potencialidad al confrontar determinadas funciones con las características de capacidad que presentan las distintas unidades ambientales para acogerlas); prognosis (para adelantar desde un primer análisis del paisaje posibles comportamientos del mismo y por tanto, dirigir dichos estudios dinámicos y empezar a pautar la sintéresis o prevención de impactos en la zona) y de sintéresis (para la prevención de impactos, sean naturales o antrópicos).

b. Determinación de los factores relacionados con la degradación ambiental (Ortiz, 2001) que involucra variables como: la determinación de la estructura espacial, los niveles de respuesta a los impactos (grados de hemerobia), medidas económicas, la degradación geoecológica, las funciones de estabilidad, mecanismos de autorregulación; las características de la organización social y los objetivos y demandas económicas. 
c. La determinación de las relaciones entre degradación y mecanismos de autorregulación (Sdasivk y Shestakov, 1994) que implican el análisis de la capacidad para asimilar medidas de prevención; el costo de las medidas de recuperación; la clasificación de los grados de degradación; la caracterización de los mecanismos de autorregulación (reversibilidad e irreversibilidad); y finalmente la determinación de áreas de autorrenovación, rehabilitación y restauración.

Las conclusiones esperadas en el trabajo de campo, deben llevar a determinar los rubros de percepción y ejecución de medidas de regulación; que de acuerdo con los autores referidos permiten:

- $\quad$ Toma de soluciones óptimas de forma operativa

- Conservar situaciones favorables bajo tendencias predominantes de degradación

- $\quad$ Corrección de una situación en estado crítico en paisajes degradados

- $\quad$ Normalizar y compensar los cambios irreversibles

- $\quad$ Pronóstico científico y toma de medidas preventivas.

- Estabilización de la situación y su sostenimiento en el contexto de un equilibrio dinámico, sobre la base de control ecológico y del pronóstico.

- Mejoramiento de la situación, hasta llevarla a un estado óptimo.

Caracterización general de la zona de estudio y ruta general

La zona de estudio comprende de manera general el paso a través de dos provincias fisiográficas: el Sistema Volcánico Transversal, La Sierra Madre Oriental y las zonas de transición entre ellas, destacándose la región de Zimapán. Las características generales de cada sector referido son:

\section{a. Sistema Volcánico Transversal}

Desde el punto de vista regional se considera como una prolongación del sistema Clarión que es afectado por la dinámica de la Placa del Caribe y la Norteamericana, la cual a su vez modifica la subducción de la Placa de Cocos y se asocia con sistemas de fallas regionales que han generado la formación de estratovolcanes, campos monogenéticos, estructuras escalonadas de tipo "Rift"; así como rasgos morfoestructurales que se reconocen 
a través de fracturas, fosas, arcos volcánicos y estructuras circulares de colapso, entre otras (Mooser el al., 1996; Ferrari el al., 2012; Capra el al., 2013).

El patrón general del sistema responde a una configuración paralela de estructuras disyuntivas en-echelon, en donde la intersección entre sistemas dominantes, permitieron el desarrollo de zonas de debilidad y la consecuente inyección de magma en un sistema de campos volcánicos poli y monogenéticos (Blatter y Hammersley, 2010; Mazzarini el al., 2010; Verma y Luhr, 2010; Cebriá el al., 2011; Lenhardt y Götz, 2011; Bernal el al., 2011; Alberico el al., 2012; Folch, 2012; Ferrari el al., 2012; Lenharrdt el al., 2013; Capra el al., 2013).

Como consecuencia de la configuración del sistema general de los morfoalineamientos locales y regionales se formaron complejos volcánicos entre los cuales se destacan: el Nevado de Colima, el Volcán de Fuego de Colima localizados en Colima y Jalisco; el volcán Ceboruco, Sanganguey y Santa María en Nayarit; el volcán Tancítaro y Paricutín en Michoacán; el Popocatépetl e Iztaccíhuatl en la cuenca de México; La Malinche en Tlaxcala, el Pico de Orizaba, Cofre de Perote y la Caldera de los Humeros en Veracruz, entre otros; campos monogenéticos como el Chichinautzin y Atlacomulco, localizados en el Distrito Federal y Estado de México de forma respectiva; así como grabens como Chapala, Silao, Querétaro y Acambay, por referir algunos ejemplos.

\section{b. Sierra Madre Oriental}

De acuerdo con Tardy et al., (1990; referido en Palacio, 1989) el origen de la Sierra se relaciona con cuatro fases que se citan textualmente:

1. Fase Cimeriana: corresponde a una transición entre fases sedimentarias y tectónicas activas, en la cual existen deformaciones poco intensas que formaron pliegues de fondo que se combinaron con largos periodos de erosión y alta productividad vegetal.

2. Fase Larámidica (FL): se presentó a finales del Paleoceno y principios del Eoceno (58 ma), y es la fase de desarrollo que se considera como responsable de estructurar a la Sierra Madre Oriental y las morfoestructuras que la conforman. En dicha etapa se presentan ajustes isostáticos regionales formados por decollements (pliegues formados por deslizamientos 
gravitacionales que involucraron con esfuerzos tectónicos comprensivos que se diferenciaron por la formación de estructuras plicativas disímiles ocasionados por las diferencias en la consistencia y cohesión litológica de los estratos, y por procesos de gravedad que experimentaron los pliegues en relación con la acumulación y disposición de materia y la pendiente regional del terreno.

Los esfuerzos predominantes tuvieron una dirección Sureste - Noreste, los cuales provocaron basculamientos positivos y deformaciones que despegaron la cobertura sedimentaria plegándola de forma independiente con referencia al basamento cristalino mesozoico.

Este proceso de "despegue" fue favorecido por estratos de lutitas formadas en alguna regresión marina (no datada), las cuales funcionaron como lubricantes que facilitaron el trabajo de la gravedad minimizando la fricción entre las rocas, generando así pliegues recostados en dirección Oeste -Este, y el predominio de laderas convexas orientadas a la vertiente del Golfo de México. Se conformó así una cadena montañosa, cuencas continentales y oceánicas; creando con ellas nuevas condiciones geográficas con campos térmicos, zonas de barlovento y sotavento y, zonas diferenciadas por humedad y radiación solar (Palacio, 1990).

De acuerdo con el autor referido, a ello se le debe de agregar el patrón climático del Eoceno que propició la formación de bosques cálidos y semi-húmedos.

Los esfuerzos tectónicos regionales combinados con los tipos y cohesión de las rocas deformadas generaron un "estrés" qué a su vez provocó la formación de detritos gruesos y finos producto de las flexiones, rupturas y fracciones entre las rocas; a los cuales se les conoce como molasas post-laramídicas. El conjunto de procesos referidos y la nueva clasificación sedimentológica de los estratos, determinan condiciones generales de inestabilidad de las vertientes y colonización vegetal en diferentes etapas.

3. Fase Miocénica (FM): esta determina la dirección final de la Sierra Madre Oriental y forma pliegues de fondo bien desarrollados que alinearon las estructuras presentes en un anticlinorio fragmentado. En segundo término, con un impulso tectónico uniforme que afectó a toda la unidad, fueron definidas las morfoestructuras del sistema serrano. 
De igual forma, Tardy (et al. 1975 pág. 10) afirma que existen evidencias de la transformación de los detritos en las cuencas y en la transición de las zonas cálido-húmedas a templadas durante el Plioceno hasta llegar a las glaciaciones del Pleistoceno.

4. Fase Pliocuaternaria: los sistemas de fallamientos conforman diferentes morfoestructuras: pliegues-bloque, pliegues mesa $\mathrm{y}$, mesas asociadas dentro de un mismo bloque. La particularidad de dicha sin clasificación en el sistema plicativo radica en que en un mismo grupo de pliegues encuentra la combinación de dichas morfoestructuras, las cuales a su vez poseen movimientos independientes.

c. Cuenca de Zimapán

Localizado en los bordes de las provincias fisiográficas de la Sierra Madre Oriental y la Mesa Central, la región posee diferentes antecedentes relacionados con la complejidad morfoestructural-estratigráfica y con la caracterización geológica-minera.

Dicha región, se convierte en el año de 2017 en el límite occidental de la Comarca Minera de Hidalgo, declarada como Geoparque por la UNESCO. Esta posee diferentes antecedentes de tipo geológico-minero y estratigráfico-estructural, que la relacionan con la provincia geológica denominada como: "Cinturón Mexicano de Pliegues y Fallas", la cual posee alta complejidad en cuanto a su génesis y evolución. En la Tabla 3 su muestra un esbozo general de las formaciones geológicas que tiene dicha región:

\begin{tabular}{c|c|l}
\hline \multicolumn{2}{|c}{ Formaciones geológico-estratigráficas } \\
\hline Terciario & Formación & \multicolumn{1}{c}{ Características distintivas } \\
\hline & Zimapán & $\begin{array}{l}\text { Se localiza dentro del anticlinal El Piñón, se caracterizó por la } \\
\text { producción de plata, antimonio, sulfuro de plomo y zinc; y en } \\
\text { dirección hacia Querétaro por mercurio. } \\
\text { Pertenece al "Tren laramídico" que tiene una dirección Noroeste - } \\
\text { Sureste que desarrolla pliegues y cabalgaduras; las fallas controlan } \\
\text { parte de la mineralización; la deformación dúctil la flora en la } \\
\text { barranca El Carrizal }\end{array}$ \\
\cline { 2 - 4 } & Lastonilco Espinas & $\begin{array}{l}\text { Se encuentra constituida por basaltos precuaternarios que forman } \\
\text { mesas }\end{array}$ \\
\cline { 2 - 4 } & $\begin{array}{l}\text { Conformada por andesitas y basaltos subyacen a la Formación El } \\
\text { Morro y son discordantes con otras formaciones del Eoceno - } \\
\text { Mioceno, se localiza en el este de Zimapán }\end{array}$ \\
\hline
\end{tabular}




\begin{tabular}{|c|c|c|}
\hline & El Morro & $\begin{array}{l}\text { Conglomerado fluvio-lacustre que afora en la cuenca } \\
\text { intracratónica de Zimapán. Se encuentra formado por calizas, } \\
\text { margas, tobas con matriz de areniscas. } \\
\text { El conglomerado se interdigita con rocas volcánicas ácidas } \\
\text { (riolitas) e intermedias (andesitas); el depósito representa una } \\
\text { molasa generada por deformaciones laramídicas. }\end{array}$ \\
\hline \multirow[t]{5}{*}{ Cretácico } & Tambara & Facie de talud \\
\hline & El Abra & Facie de plataforma \\
\hline & El Doctor & Facie de plataforma \\
\hline & Tamaulipas & Facie de cuenca \\
\hline & Sovatal & $\begin{array}{l}\text { Secuencia marina tipo flysch de lutitas, margas y calizas alternadas } \\
\text { con estratos formados por pliegues sinsedimentarios y estructuras } \\
\text { nodulares. }\end{array}$ \\
\hline Jurásico & Las Trancas & $\begin{array}{l}\text { Conformada por lutitas calcáreas, limolitas, calizas con pedernal, } \\
\text { grauvaca y areniscas. Aflora en ella el núcleo del anticlinal } \\
\text { Bonanza y el anticlinorio El Piñón a lo largo del río Extorax, es } \\
\text { discordante con la Formación El Doctor, El Abra y Tamaulipas. } \\
\text { Se desarrolla por debajo de la Caliza Tamaulipas que posee } \\
\text { calizas, margas y lutitas apizarradas. }\end{array}$ \\
\hline
\end{tabular}

Tabla 3. Formaciones geológicas pertenecientes a la cuenca de Zimapán. Fuente: elaboración propia con base en: Carrillo, 1971; Carrillo y Suter, 1982; Navarro, 1982; et al., 1992; Corona y Carrillo, 1997; Ortega et al., 1992; Mascuraño et al., 2013.

Descripción general de sitio y actividades

La Figura 1 muestra la ruta de la práctica de campo, saliendo desde la ciudad de Toluca y planeada para un recorrido de tres días.

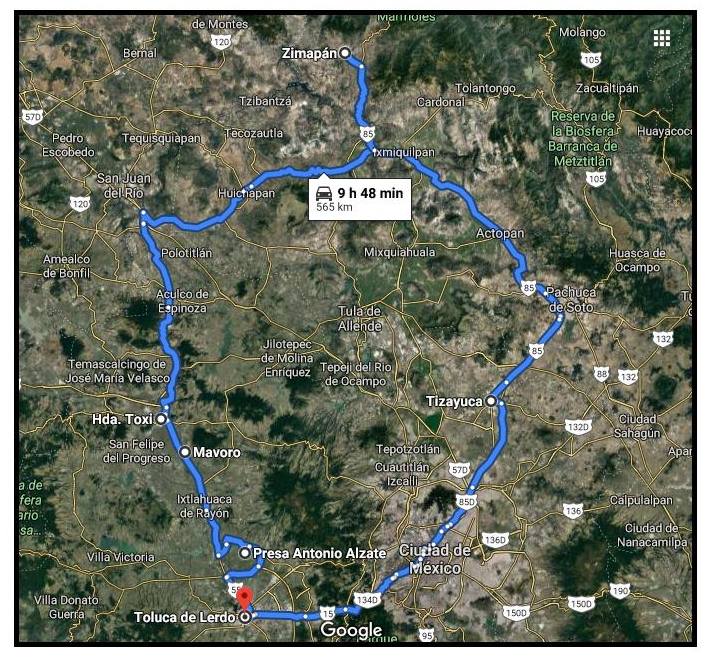

Figura 1. Ruta general de campo. Fuente: Elaboración propia con base en Google maps; trazado en diciembre de 2019. 


\section{Parada 1. Ranchería San José Las Lomas}

El sitio es característico por encontrarse en una zona de lomeríos a los cuales debe su nombre; el origen geológico y geomorfológico de este sitio se debe a los diferentes procesos relacionados con el graben de Toluca-Ixtlahuaca y a los procesos de distención que forman estructuras en fosa y, bloques basculados tectónicamente.

Los procesos exógenos que conforman el lugar es la existencia de cárcavas escalonadas características por el cambio de material de los estratos ya que cada uno contiene diferentes tipos de roca y materiales más deleznables que de otros estratos haciendo que la ley geomorfológica de "mayor pendiente mayor- erosión no se cumpla", creando así el tipo de erosión aereolar, la cual es característica porque en la zona existe arena, limo y arcilla sumando la agricultura del lugar y la existencia de ganado que deterioran con el tiempo el suelo.

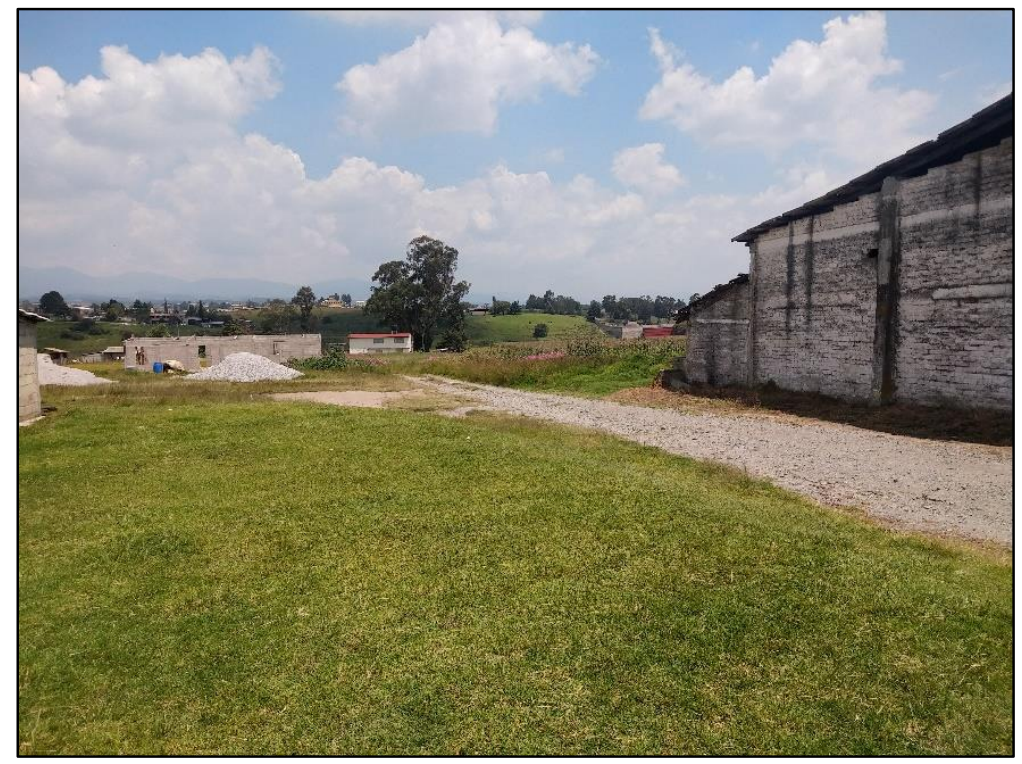

Figura 2. Panorámica del rancho San José en las proximidades de Taborda, Estado de México. Fuente: fotografía del autor obtenida en septiembre de 2019.

Actividades realizadas y sugeridas: Observación general del paisaje, descripción geográfica del entorno geográfico y clasificación geomorfológica del relieve. Determinación de procesos erosivos (cárcavas) y zonas susceptibles a la degradación. 
Determinación geomorfológica de valles asimétricos, sistemas de drenaje pinado y asimetría de valles por influencia de basculamientos regionales asociados a la fosa Toluca - Ixtlahuaca.

Parada 2. Presa José Antonio Álzate

La presa Álzate se encuentra contaminada tanto con aguas negras que llegan de la ciudad de Toluca y por concentraciones de basura que cubren las márgenes y canales de crecidas del río Lerma.

Se advierte el uso de agua para la agricultura en donde los cultivos adaptados a este entorno, generan alimentos que se venden en pueblos cercanos y se desechan en el entorno, generando así condiciones de entropía ambiental.

Se observa que el filtro y los productos químicos empleados para purificar el agua resultan insuficientes y que el entorno en general es tóxico; así que el impacto en general es alto.

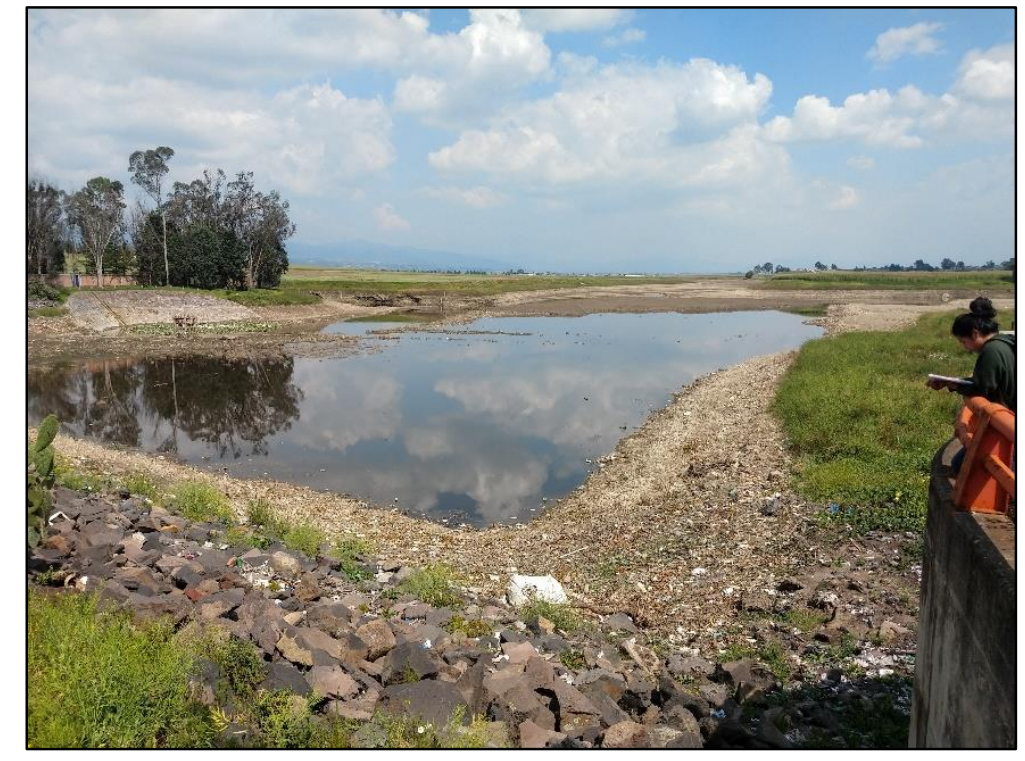

Figura 3. Cortina de la presa Juan Antonio Alzate, Estado de México. Fuente: fotografía del autor obtenida en septiembre de 2019. 
Actividades realizadas y sugeridas: se realiza la caracterización biótica y abiótica del paisaje; determinación de feno y cripto sistemas. Se establecen criterios en campo para la conformación de Unidades Ambientales y se propone la aplicación de índices físicogeográficos, socioeconómicos y paisajísticos.

En dicho sitio se aplican exámenes de observación y análisis de componentes del paisaje; así como de la estructura vertical y horizontal del paisaje.

Parada 3. Carretera Toluca-Atlacomulco; desviación a Jocotitlán

En este punto se observa el volcán Jocotitlán advirtiendo la morfología asimétrica y acumulativa que se relaciona con actividades eruptivas explosivas. Se logró hacer un ejercicio de reconstrucción a través de la geoforma denotándose la morfología del cráter destruido, un sistema de transcurrencias y coladas lávicas fisurales; advirtiéndose la formación y la morfología de hummocks al norte del edificio.

Se realizan observaciones referentes a la densidad de vegetación y estado que guarda en el contexto general de las geoformas y el uso de suelo. Se destaca la correlación productividad agropastoril, calidad y temperatura del agua.

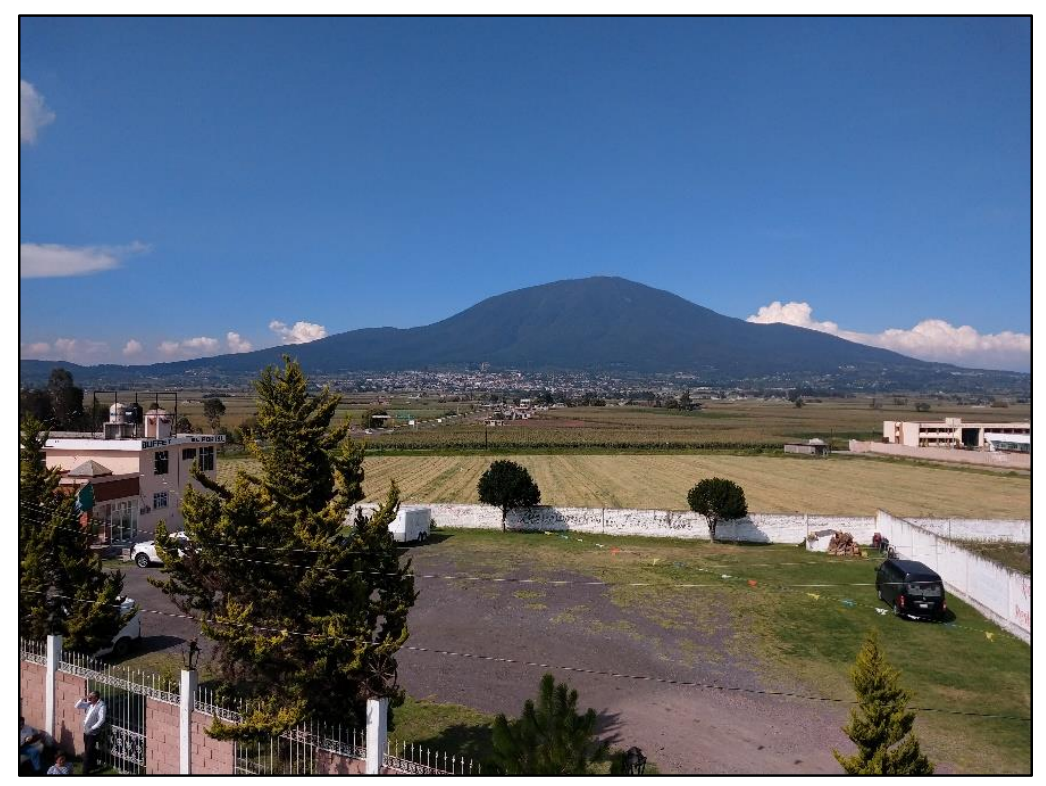

Figura 4. Vista del volcán Jocotitlán, Estado de México. Fuente: fotografía del autor obtenida en septiembre de 2019. 
Actividades realizadas y sugeridas: se realiza un levantamiento geomorfológico y se realiza la interpretación de unidades de relieve; correlación con el sistema abiótico y con las actividades socioeconómicas. Se hace referencia a condiciones relacionadas con riesgos (vulnerabilidad, exposición) y se aplica la Ecuación General de Riesgo (EGR; Espinosa y Hernández; 2015 y 2018) para la evaluación de variables correlacionadas.

\section{Parada 4. Falla de Toxi}

Corresponde a un bloque hundido asociado a la falla de Toxi (Falla Pastores); la morfología del sistema disyuntivo presenta componentes verticales y horizontales, y la deformación en el espejo de falla indica condiciones de compresión. Se explica el principio geomorfológico desarrollado en el aula que establece "Entre más sinuosa más peligrosa" acotado por el profesor responsable de la práctica.

Este lugar tiene una importancia geológica y geomorfológica muy particular ya que aquí se encuentran diatomeas aflorando a más de 40 metros sobre el nivel de base, se encuentran diaclasas y fallas con movimientos verticales y horizontales; así como micropliegues en estratos paralelos de sedimentos que presentan disyunciones.

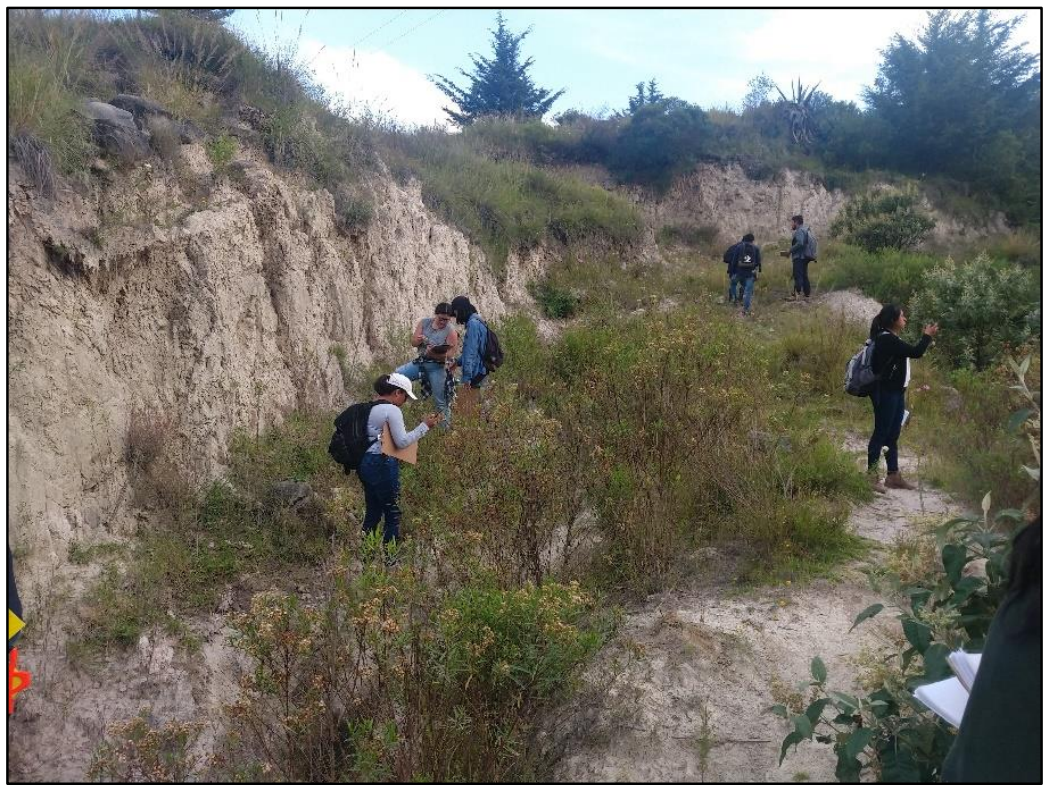

Figura 5. Depósitos de diatomitas en Toxi, Estado de México. Fuente: fotografía del autor obtenida en septiembre de 2019. 
Sin embargo, por encima de este estrato se encuentra un derrame de basalto, indicador de que primero existió el lago, posteriormente hubo un evento volcánico y finalmente, ascensos tectónicos locales y regionales diferenciales.

Actividades realizadas y sugeridas: Determinación regional de los paisajes; interpretación de las deformaciones tectónicas del paisaje del sistema disyuntivo (vertical y horizontal); correlación con el sistema Acambay - Morelia y Complejo volcánico de Temascalcingo. Se realiza un ejercicio sobre columnas estratigráficas, medición de isopacas y altura de elementos morfológicos y bióticos (árboles).

Parada 5. San Juan del Rio, carretera de Ixquimilpan

Zona límite de la caldera volcánica de Huichapan, corresponde a la región de transición entre provincias fisiográficas de la Mesa del centro y el Sistema Volcánico Transversal, en la cual se presentan fallas con dirección NE y SE, así como derrames fisurales de andesitas y basaltos, además de afloramientos de tobas y riolitas.

El tipo de vegetación es xerófila, además por erosión se presenta cárcavas de $1 \mathrm{~m}$ de profundidad en donde, el suelo tiende a presentar poco desarrollo debido a afloramiento de las rocas y la alta pedregosidad. Se denota control estructural paralelo a través del parteaguas local, los lineamientos desarrollados sobre el sistema de laderas y el cauce encajado del río principal.

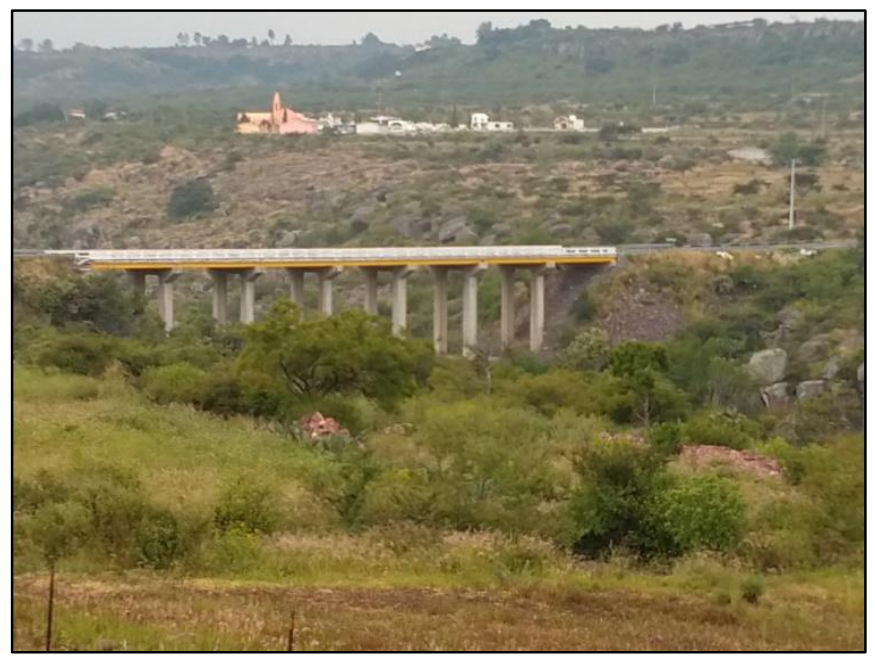


Figura 6. Paisaje correspondiente a la barranca localizada en la carretera San Juan del Rio Ixquimilpan. Fuente: fotografía del autor obtenida en septiembre de 2019.

Actividades realizadas y sugeridas: Conformación de unidades de paisaje; determinación de lineamientos y sistemas de control estructural y litológico. Clasificación de las comunidades vegetales y determinación de las características del suelo

Punto 6. Huichapan (Autopista)

Esta zona se encuentra en el borde la de la caldera de Huichapan, la cual solo quedan vestigios debido a que la propia actividad explosiva y la erosión la han destruido. Ésta puede ser considerada para un geositio. Se encuentra entre dos provincias fisiográficas, la Madre Sierra Oriental y la Mesa Central. Este tipo de formación se generó en cuatro etapas:

1. Caldera de forma circular con basamentos de más de 11 millones años

2. Explosión piroclástica, la cual está activa por la cámara magmática que sigue emitiendo material

3. Desarrollo de actividad piroclástica y destrucción por erosión

4. Se reactiva por la caldera y se forma un campo monogenético

Para conocer más acerca de este sitio, se aplica un formato de valor intrínseco, en el cual se requería una observación detallada en la zona y región. Según la geología, esta zona está conformada por dacita, riolita y andesita. Actividades realizadas y sugeridas: Se hace un levantamiento completo de unidades de paisaje y se aplican índices relacionados con geodiversidad, geoconservación y geoparques para determinar la riqueza patrimonial geológica, geomorfológica y de paisaje.

Parada 7. Presa de Zimapán

Refiere a una zona de cañones y valles anticlinales formados por procesos plicativos, los cuales a su vez, se encuentran afallados, motivo por el cual los estratos de roca se encuentran discordantes y, a su vez, presentan evidencias de erosión intensa y la presencia de un dique que implica el desarrollo de procesos metamórficos de contacto que se relacionan 
regionalmente con los yacimientos de placer productores de oro y zinc, los cuales se extienden a Querétaro.

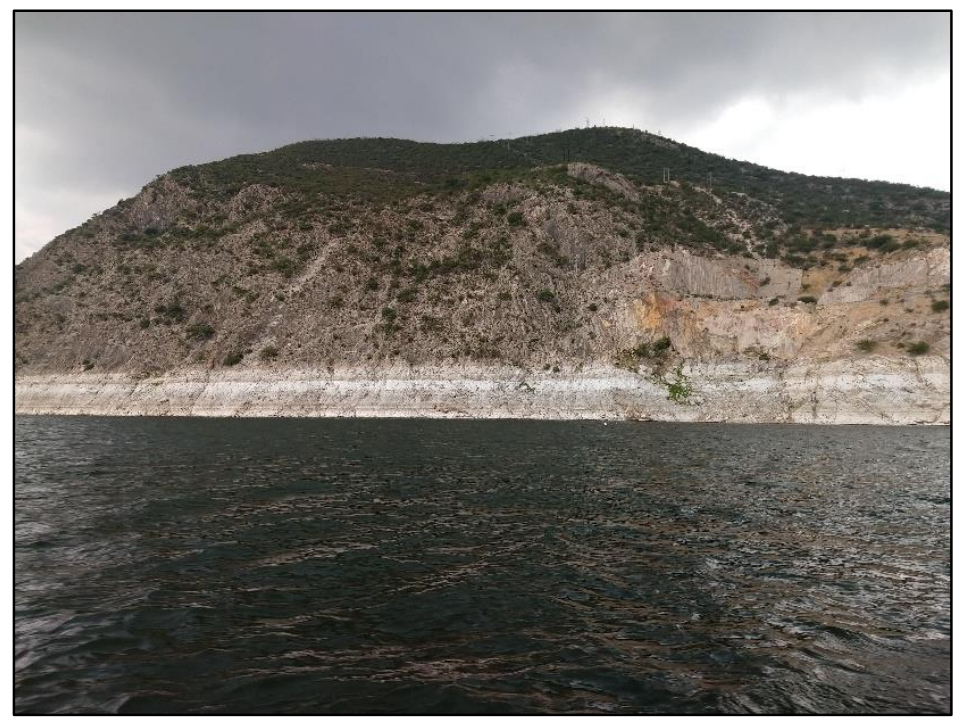

Figura 7. Afloramientos de calizas en la presa de Zimapán; límite estatal entre Hidalgo y Querétaro. Fuente: fotografía del autor obtenida en septiembre de 2019.

La presa conecta a los ríos Moctezuma y San Juan en el sistema Pánuco y fue construida para generar energía eléctrica para abastecer a los estados de Querétaro, Hidalgo, México y la Ciudad de México. Cuenta con una profundidad de 205 metros y una extensión de $45 \mathrm{~km}$., ayuda con el control de crecidas extraordinarias y favorece el sistema de riego en la región conocida como Valle del Mezquital; recibe aportes de la ciudad de México.

Actividades realizadas y sugeridas: Análisis de la configuración del relieve, distinción de los sistemas plicativos y disyuntivos de la región, así como del metamorfismo y descripción de las formas de erosión-acumulación. Se realizan ejercicios relacionados con prognosis, diagnosis y sintéresis del paisaje y se contrastan con los beneficios de uso de suelo y socioeconómicos.

Parada 8. Pacula 
Se observan formaciones de cárcavas por escurrimientos y erosión fluvial, algunas de ellas son de forma rectangular alargada y otras son de escalonamiento, la primera se forma porque el tipo de material es deleznable, y la segunda porque la biota interviene en su formación, se presentan deformaciones en el terreno conocidas como "pie de vaca", provocadas por el tránsito continuo de ganado en las laderas. El material poco consolidado, las condiciones climáticas, meteorológicas y las actividades agropastoriles los animales se conjugan para la formación cárcavas.

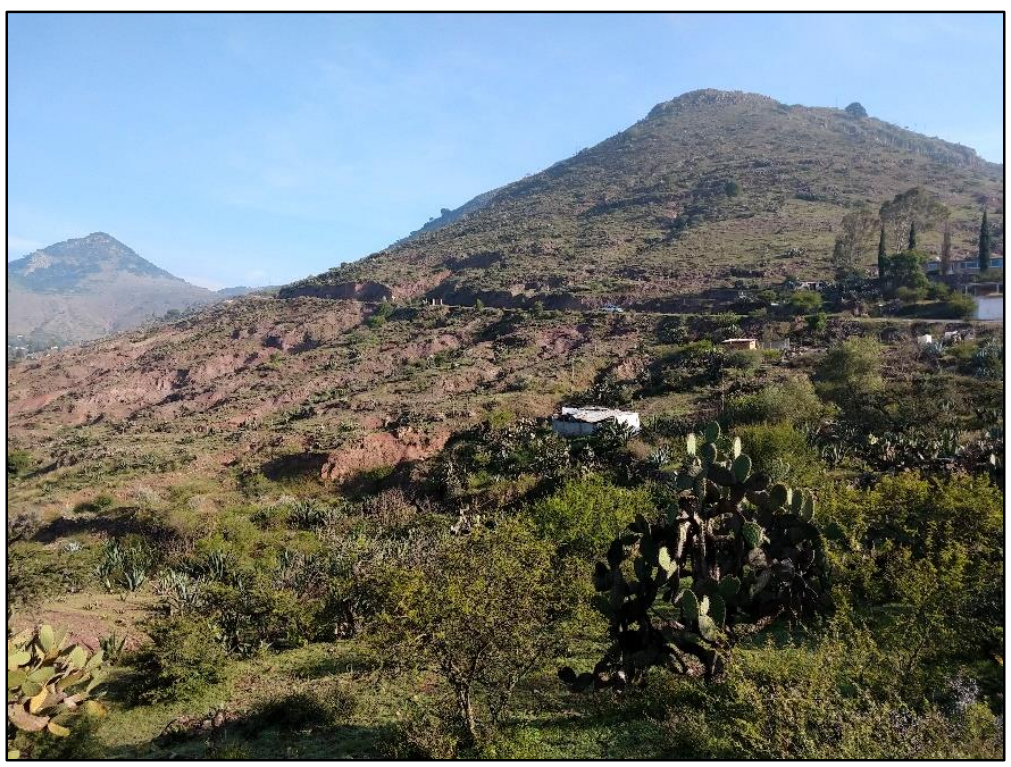

Figura 8. Sistema erosivo de cárcavas en Pacula, Estado de Hidalgo. Fuente: fotografía del autor obtenida en septiembre de 2019.

Actividades realizadas y sugeridas: En este sector se observa la forma de estructuración de los estratos vegetativos y se correlaciona con la estabilidad del paisaje, se identificaron indicadores ambientales de degradación como la presencia de epífitas invasoras, desarrollo de vegetación secundaria de primera y segunda generación, así como de la inserción ambiental de moscos por cambio de temperatura.

Parada 9. Ciudad Valles 
Se observó la formación de cárcavas a mitad de ladera influenciadas por la presencia de fracturas. La vegetación observada era secundaria y sobre ella se denotan procesos de deforestación; así mismo se advierte que en la reforestación se introdujo eucalipto a pesar de que es una planta invasora y agresiva porque roba el agua de otras plantas y que su olor puede ser toxico para otras especies.

La zona pertenece a la Formación Las Trancas perteneciente a la era Jurásica y esta se relaciona con la era Cretácica; en ella se encuentran depósitos flysch; así mismo se encuentran afloramientos y formaciones como "El Morro" formada por conglomerados y molasas, y la formación "Atotonilco", de comportamiento basáltico y perteneciente al periodo Cuaternario.

Actividades realizadas y sugeridas: Se introdujeron los patrones de clasificación referentes a modelos de conservación y geoconservación; se aplica los conceptos morfoedáficos (Espinosa, 2009) y se distinguen problemas-propuestas de solución a tópicos relacionados con asimilación antrópica, porcentaje y estado de la cobertura vegetal.

Parada 10. Parque Nacional de los Mármoles. Punto 1

Se observan partes de un plegamiento metamorfizado regionalmente, relieve invertido, horizontes del suelo delgados, y selva media. Los escarpes están caracterizados por intemperismo en rocas de color rojo (oxidación de minerales hierro).

El área se considera relativamente como un "Paisaje Prístino" en el tiempo actual. 


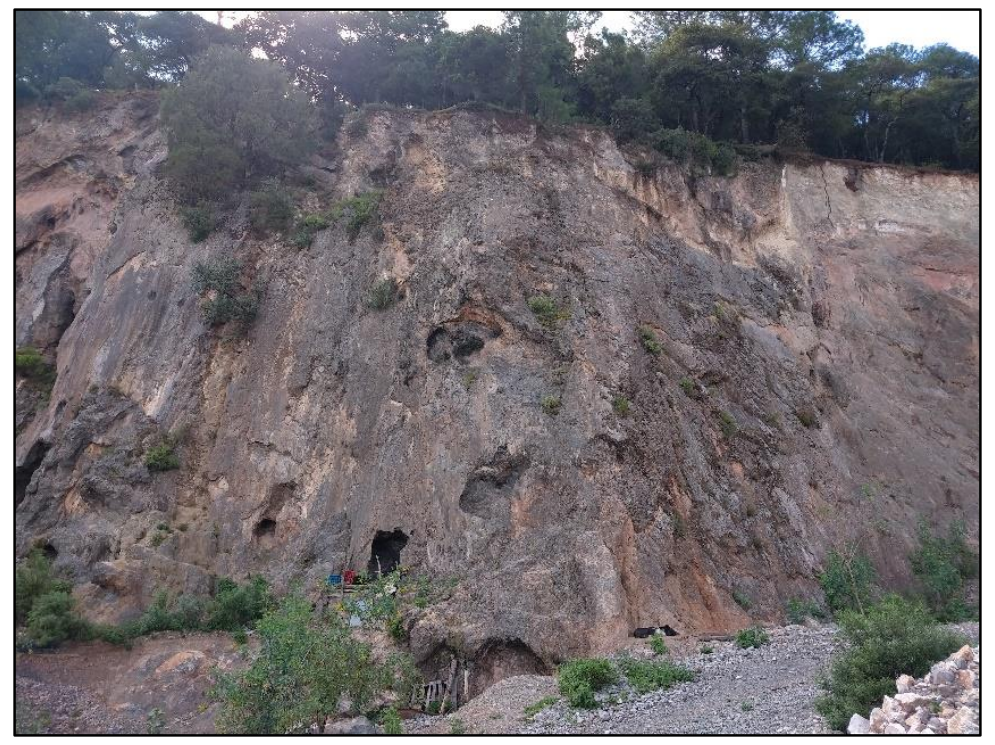

Figura 9. Afloramiento de mármol al interior del Parque Nacional de los Mármoles. Estado de Hidalgo. Fuente: fotografía del autor obtenida en septiembre de 2019.

Actividades realizadas y sugeridas: Se realiza la caracterización del perfil estratigráfico y correlación geológica, geomorfológica y paisajística en el contexto regional. Se incluye el análisis histórico regional contrastado con la legislación mexicana vigente.

Parada 11. Parque Nacional de los Mármoles. Punto 2

Se observan los escarpes y valles formados en la zona de transición entre la Sierra Madre Oriental y el Sistema Volcánico; se encuentra dominado por metamorfismo regional, el cual responde a la formación del mármol y de las actividades de extracción.

Asimismo se encuentran evidencias de metamorfismo de contacto en donde los diques formaron cristalizaciones y la consecuente formación de placeres y otros elementos como el oro, la plata, el mercurio y el zinc. Actividades realizadas y sugeridas: Aplicación de índices de carácter biótico, abiótico, socioeconómico y paisajístico de la región. Integración e interpretación de resultados.

Se propone la realización de una evaluación de desempeño y aprendizaje de los estudiantes; y una de retroalimentación general. 


\section{Conclusiones}

La región comprendida entre los Estados de México, Hidalgo y Querétaro presenta características particulares relacionadas con un complejo bosquejo geológicogeomorfológico que correlaciona elementos plicativos, disyuntivos, erosivos y deposicionales que han interactuado con elementos de orden climático y meteorológico que en conjunto, otorgan a la región un legado histórico de ocupación-explotación y conservación en el tiempo presente.

El has de relaciones que se genera en torno a los elementos del paisaje, otorgan al estudiante de la Licenciatura en Geografía un escenario ideal para el desarrollo de habilidades y el incremento de ellas de manera progresiva según avanza a lo largo del curso y, conforme se gesta un proceso de maduración cognitiva durante el trabajo de campo; el cual como se advierte; el orden de complejidad se incrementa conforme se recorre la zona de estudio y aplican técnicas y métodos para el análisis del espacio geográfico desde la perspectiva del Paisaje, para con ello argüir los resultados encontrados; y con ello fijar posturas en torno a temas de ordenamiento territorial, riesgo, equilibrio y balance morfoedáfico, impacto ambiental y otros que son acordes con los objetivos de carrera y el perfil profesional deseado.

\section{Referencias bibliográficas}

Alberico I., Petrosino P., Mglione G., Bruno L., Capaldo F., Dal A., Lirer L. and Mazzola S. (2012). Mapping the vulnerability for evacuation of the Campi Flegrei territorial system in case of a volcanic unrest. Natural Hazardas (64): 1823-1854

Bernal J., Lachniet M., McCulloch M., Mortimer G., Morales P. and Cienfuego E. (2011). A speleothem record of Holocedne climate variability from southwestern Mexico. Quaternary Research 75 (2011): 104-113.

Blatter W. and Hammersley L. (2010). Impact of the Orozco fracture zone on the central Mexican Volcanic Belt. Journal of Volcanology and Geothermal Research, Elsevier 197. (2010): 67-84. 
Capra L., Bernal J., Carrasco G. and Roverato M. (2013). Climatic fluctuations as a significant contributing factor for volcanic collapses. Evidence from Mexico during the Late Pleistocene. Global and Planetarry Change 100 (2013): 194-203.

Carrillo J. (1971). La plataforma de Valles - San Luis Potosí. Boletín de la Asociación Mexicana de Geólogos Petroleros 23(1): 102.

Carrillo M. y Suter M. (1982). Tectónica de los alrededores de Zimapán, Hidalgo y Querétaro. Sociedad geológica Mexicana. Libro guía de la excursión geológica a Zimapán y áreas circundantes 1(1):1-20.

Cebriá J., Martín C., López J. Morán D. and Martiny B. (2011). Numerical recognition of alignments in monogenetic volcanic areas: examples from the MichoacanGuanajuato Volcanic Field in Mexico and Calatrava in Spain. Journal of Volcanology and Geothermal Research, Elsevier. 201. (1-4): 73-82.

Corona R., Carrillo M. (1997). Control estructural y rasgos geomorfológicos relacionados con la mineralización de la región comprendida entre Zimapán Hidalgo y San Joaquín, Querétaro. Trabajos Técnicos, Geología, Minería, Metalurgia, Temas generales. Asociación de Ingenieros de Minas, Metalurgistas de México A.C. 1(1): 19.

Espinosa L. 2009. Metodología para la evaluación morfoedáfica en sistemas de laderas en zonas templadas. Ciencia Ergo-Sum. Ciencias de la Tierra y de la Atmósfera. Vol. 16. No. 3. Noviembre 2009-Febrero 2010. ISSN 1405-0269.

Espinosa L. y Hernández J. 2015. Estudio del riesgo. Análisis multifactorial, multinivel y multitemporal. En: Revista Latinoamericana el Ambiente y las Ciencias. 6(14):1-27 ISSN: 2007-512X

Espinosa L. y Hernández J. 2018. Ecuación general de riesgo (EGR): principios generales. En: Enfrentando los riesgos socionaturales. J. Baró y F. Monroy Eds. Universidad Politécnica de Cuautitlán Izcalli. México, ISBN 978-607-457-5. Pp 309-330. 
Ferrar, L., Orozco T., Manea V. and Manea M. (2012). The dynamic history of the TransMexican Volcanic Belt and the Mexico subduction zone. Journal of Volcanology and Geothermal Research, Elsevier. 522-523 (2012): 122-149.

Folch A. (2012). A review of tephra transport and dispersal models: Evolution, current status and future perspectives. Journal of vulcanology and geotermal research. Elsevier 235236 (2012): 1-14.

Lenhardt N. and Götz A. (2011). Volcanic settings and their reservoir potential: An outcrop analog study on the Miocene Tepoztlán Formation, Central Mexico. Journal of Volcanology and Geothermal Research, Elsevier. 204. (2011): 66-75.

Lenharrdt N., Böhnel H., Hinderer M. and Hornung J. (2013). Paleocurrent direction measurements in a volcanic setting by means of anisotropy of magnetic susceptibility: a case study from the Lower Miocene Tepoztlán Formation (Transmexican Volcanic Belt, Central Mexico). Sedimentary Geology. 1(2013): 1-14.

Mascuraño E., Levrese G., Cardellach E., Tritlla J., Corona R., Meyzen C. (2013). Postlaramide, Eocene magmatic activity in Sierra Catorce, San Luis Potosi, Mexico. Revista mexicana de Ciencias geológicas 30(2): 299-311.

Mooser F., Montiel A. y Zúñiga A. (1996). Nuevo mapa geológico de las cuencas de México, Toluca y Puebla. Estratigrafía, tectónica regional y aspectos geotérmicos. Comisión Federal de Electricidad. 68 p.

Navarro R. (1982). Geología y operación minera del cuerpo Concordia NW, El Monte, distrito de Zimapán, Estado de Hidalgo. Comisión Federal de Electricidad. Libro guía de la excursión geológica a la región de Zimapán y áreas circundantes; estados de Hidalgo y Querétaro 1(1): 28-32.

Ortega G., Mitre L., Roldán J., Arana J., Morán D., Alanís S., Nieto A. (1992). Carta geológica de la República Mexicana, escala 1:2,000,000 y texto explicativo. Instituto de Geología, UNAM, Secretaría de Energía, Minas e Industria Paraestatal, Consejo de Recursos Naturales 1(1): 74. 
Palacio G. (1989). Breve análisis geomorfológico ambiental en las márgenes del río NecaxaTecolutla. Tesis de Licenciatura en Geografía. Facultad de Filosofía y Letras. Universidad Nacional Autónoma de México. 108 p.

Tardy M., Longoria J., Martínez J., Mitre L., Patiño M., Podilla R., Ramírez C. (1975). Observaciones generales sobre la estructura de la Sierra Madre Oriental: la aloctonía del conjunto cadena alta-altiplano central en Torreón, Coahuila y San Luis Potosí. Revista del Instituto de Geología, UNAM 1(1):1-11.

Verma S. and Luhr, J. (2010). Sr, $\mathrm{Nd}$ and $\mathrm{Pb}$ isotropic evidence for the origin and evolulution of the Cántaro-Coplima volcanic chain, Western Mexican Volcanic Belt. Journal of Volcanology and Geothermal Research, Elsevier. 197 (1-4): 33-51. 